\title{
Septic pulmonary emboli as a complication of peripheral venous cannula insertion
}

\author{
Ankita Baidya', Vanishri Ganakumar', Ranveer S Jadon', Piyush Ranjan ${ }^{1, *}$, \\ Smita Manchanda ${ }^{2}$, Rita Sood ${ }^{1}$ \\ ${ }^{1}$ Department of Medicine, All India Institute of Medical Sciences, New Delhi, India; \\ ${ }^{2}$ Department of Radiodiagnosis, All India Institute of Medical Sciences, New Delhi, India.
}

\begin{abstract}
Summary Septic pulmonary emboli can occur as a complication of many diseases, most common being right sided infective endocarditis. Septic emboli through a peripheral venous cannula are rarely reported in literature though central venous catheter is commonly implicated. We describe a case of widespread cellulitis and septic pulmonary emboli as a complication of peripheral venous cannulation.
\end{abstract}

Keywords: Cellulitis, septic emboli, cannulation

\section{Introduction}

Septic embolism can have varied presentations and clinical considerations. The major complication is due to vascular occlusion of the involved tissues or organs. Infected central venous catheters are commonly associated with septic emboli but peripheral vascular catheters are rarely implicated. We describe a rare case of septic pulmonary emboli related to infected peripheral venous cannulation caused by an unusual etiological agent.

\section{Case Report}

A 26-year-old gentleman of Uttarakhand, India, presented with complaints of fever, productive cough, sudden onset shortness of breath and cellulitis in both the upper limbs. He had been recently hospitalized for dengue fever and administered intravenous fluids through peripheral venous line. On physical examination, patient was febrile, tachypneic and in respiratory distress. On local examination, there were multiple pus filled bullae in left hand and swelling and erythema involving right forearm that had started at the site of cannulation. Chest examination showed active

*Address correspondence to:

Dr. Piyush Ranjan, Associate Professor, Department of Medicine, All India Institute of Medical Sciences, Ansari Nagar, New Delhi-110029, India.

E-mail: drpiyushdost@gmail.com accessory muscles of respiration, stony dull percussion at the base of right lung and decreased breath sounds at right infrascapular, infraaxillary and mammary area. Other system examination was unremarkable. Chest $\mathrm{X}$-ray revealed bilateral multiple patchy heterogeneous peripheral opacities and infiltrates with right sided pleural effusion. Contrast enhanced computed tomography (CECT) chest showed feeding vessel sign confirming the diagnosis as septic emboli (Figure 1). Venous Doppler and 2D-echocardiogarm were normal.

Laboratory findings showed marked leukocytosis $\left(22,000 / \mathrm{mm}^{3}\right)$. Pus aspirate, blood sample and sputum sample were sent for microbiological testing. Patient was started empirically on ceftriaxone, vancomycin and clindamycin. The pus culture and sputum culture showed Klebsiella pneumoniae sensitive to cefoperazone-sulbactum, piperacillin-tazobactum, meropenem and amikacin. The antibiotics were modified accordingly to antimicrobial sensitivity profile to cefoperazone-sulbactum. Despite giving appropriate antibiotics, he continued to have fever though intensity of fever declined. Bronchoalveolar lavage (BAL) was done to confirm the etiology. The BAL sample was sent for galactomannan, fungal $\mathrm{KOH}$ mount and culture, AFB stain, geneXpert, gram stain, bacterial culture and sensitivity. BAL galactomannan, geneXpert and fungal culture were negative. BAL culture showed Klebsiella pneumoniae with same antimicrobial sensitivity profile. Diagnostic pleural tap was done. Laboratory findings of pleural fluid showed occasional lymphocytes in proteinaceous background. AFB stain, geneXpert and 


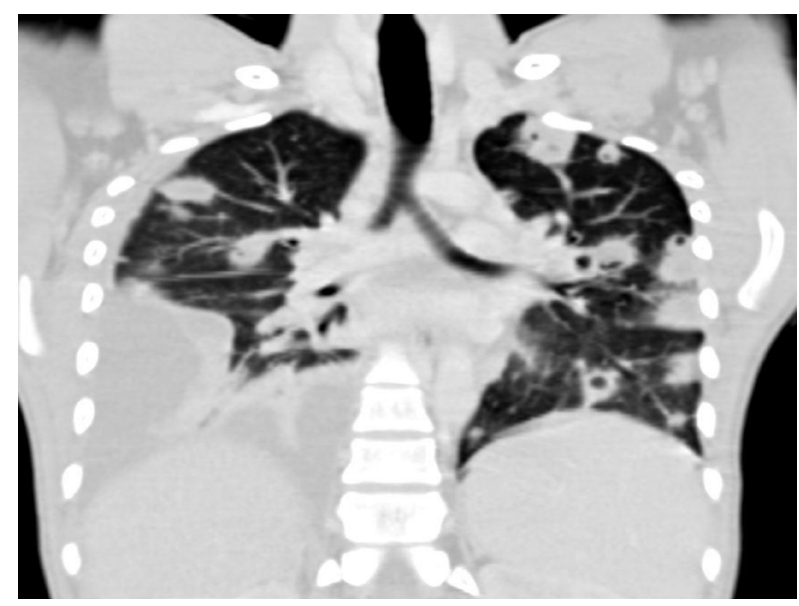

Figure 1. Contrast enhanced computed tomography chest image showing feeding vessel sign confirming the diagnosis as septic emboli

culture were sterile. On day 6 of starting cefoperazonesulbactum, patient became afebrile. The skin lesions improved significantly. He was administered 2 weeks of cefoperazone-sulbactum and discharged on oral faropenem for 4 weeks. At the time of discharge, total leukocyte count (TLC) was $11,200 / \mathrm{mm}^{3}$ with marked radiological resolution of lung opacities and healed skin lesions. He was followed up regularly. Chest X-ray and skin lesions showed complete resolution after 8 weeks.

\section{Discussion}

Catheter related blood stream infections are usually reported due to central venous catheters. Other complications seen due to venous catheters are thrombophlebitis, cellulitis, septic thrombophlebitis rarely tissue necrosis and septic emboli (1). Septic emboli refer to embolization of infectious particles in the circulation. Depending upon the site of embolization, the clinical presentation can vary.

There are various routes related to infection of peripheral venous catheter. These are extraluminal, intraluminal, hematogenous spread, and use of contaminated infusate (2). Extraluminal route is most common and infecting microorganisms are usually endogenous skin flora predominantly composed of gram positive bacteria. The skin organisms migrate through the narrow extraluminal space created due to insertion of intravenous catheter. Contamination of catheter hub can occur either due to skin organisms or inappropriate hand hygiene of healthcare worker. A study done by Lee WL et al. (3) found that continuous intravenous infusion more than 24 hours, use of infusion pumps, insertion of cannula in lower extremity and any pre-existing neurological and neurosurgical sequelae are independent risk factors that increase the chance of soft tissue infections related to peripheral intravenous cannulation.

The usual microorganisms in catheter related infections are gram positive bacteria accounting for almost two-third of cases. Among all, Staphylococcus aureus is the commonest. Pseudomonas aeruginosa is the most frequently grown gram negative bacteria from such infections, almost always associated with inappropriate health care practices $(4,5)$. In the present case, the etiological agent was extended spectrum betalactamase (ESBL) producing Klebsiella pneumoniae, an uncommon pathogen in such clinical presentation of cellulitis and septic pulmonary emboli. This drug resistant organism might have been acquired due to improper hand hygiene of health care worker.

Till date only couple of cases of septic pulmonary emboli through peripheral intravenous catheter have been reported in English literature. First case was reported by Friedberg et al. (6) from Australia. A patient of schizophrenia with altered sensorium had multiple septic emboli who succumbed to his illness. Another report is by Fidan et al. (7) from Turkey. The patient had septic embolus and recovered on intravenous antibiotics. In present case, the patient was an apparently healthy male, who developed this complication and recovered completely after 6 weeks of intravenous antibiotics.

This clinical entity is commonly seen in patients suffering from right sided bacterial endocarditis, infected central venous catheters, periodontal infection and prosthetic vascular devices. The most common symptom is fever (93\%) followed by chest pain, respiratory distress, cough and hemoptysis (8). Respiratory symptoms are present in less than $50 \%$ of cases of septic pulmonary emboli and so demand a high index of clinical suspicion to promptly administer intravenous antibiotics for good prognosis.

This case highlights that a simple procedure of peripheral intravenous cannulation can lead to catastrophic complication of septic pulmonary emboli and widespread cellulitis if not done with proper care and precautions. Also the usual pathogens in such clinical settings are gram positive bacteria, but with the history of recent hospitalization empirical therapy should also cover drug resistant gram negative microorganisms. It also emphasizes the importance of appropriate healthcare practices to be taken care during all procedures.

\section{References}

1. Stawicki PS, Firstenberg MS, Lyaker MR, Russell SB, Evans DC, Bergese SD, Papadimos TJ. Septic embolism in intensive care unit. Int J Crit Illn Inj Sci. 2013; 3:58-63.

2. Cmich CJ, Maki DG. The promise of novel technology for prevention of intravascular device-related bloodstream infections I: Pathogenesis and short-term use devices. Clin Infect Dis. 2002; 34:1232-1242.

3. Lee WL, Liao SF, Lee WC, Huang CH, Fang CT. Soft tissue infections related to peripheral intravenous catheters in hospitalised patients: a case-control study. J Hosp Infect. 2010; 76:124-129. 
4. Gahlot R, Nigam C, Kumar V, Yadav G, Anupurba S. Catheter-related bloodstream infections. Int J Crit Illn Inj Sci. 2014; 4:162-167.

5. Parameswaran R, Sherchan JB, Varma MD, Mukhopadhyay C, Vidyasagar S. Intravascular catheterrelated infections in an Indian tertiary care hospital. J Infect Dev Ctries. 2011; 5:452-458.

6. Freiberg DB, Barnes DJ. Fatal sepsis following peripheral intravenous cannula embolus. Chest.1992; 101:865-866.
7. Fidan F, Acar M, Unlu M, Cetinkaya Z, Haktanir A, Sezer M. Septic pulmonary emboli following infection of peripheral intravenous cannula. Eur J Gen Med. 2006; 3:132-135.

8. Ye R, Zhao L, Wang C, Wu X, Yan H. Clinical characteristics of septic pulmonary embolism in adults: A systematic review. Respir Med. 2014; 108:1-8.

(Received April 6, 2018; Revised April 25, 2018; Accepted April 28, 2018) 